\title{
Modeling Measurement Uncertainty in Room Acoustics
}

P. Dietrich

This paper investigates a way of determining and modeling uncertainty contributions in measurements of room acoustic parameters, which are commonly used to describe the acoustic situation of a room in an objective manner. If the range of uncertainty and the confidence interval are not given, the results remain incomparable to other measurement teams, since modern PC-based measurements still show appreciable sources of measurement errors. The Guide to the Expression of Uncertainty in Measurement (GUM) defines a unified guideline for determining uncertainties in all fields of measurement. Its application is increasingly required by modern measurement standards. However, the GUM procedures have not been applied to room acoustics yet. Hence, a scalable linear approach for calculating the combined uncertainty of room acoustic parameters with regard to the input quantities is proposed. In-situ measurement results of specially designed experiments show the significance of the main influence factors and are used to build the uncertainty budget.

Keywords: Room acoustics, measurement uncertainty, GUM, ISO 3382, uncertainty modeling.

\section{Introduction}

Room acoustic parameters are commonly used to describe a room's acoustical quality in a simplified and objective manner, where the most common parameter is the reverberation time [1]. Usually, a personal computer and professional audio hardware, meeting certain requirements, are essential for these measurements, as defined in ISO 3382 [2] and ISO 18233 [3]. By comparing the measurement results obtained by different teams for the same room, deviations larger than the human perception have been found [4]. A good measurement accounts for a range of uncertainty smaller than this perception, to obtain parameters corresponding to the listener's impressions in a room. Furthermore, world-wide round robins have been conducted in the past to investigate these variations and their significance [5].

In order to provide unified results in all fields of measurement, the ISO/BIPM developed the Guide to the Expression of Uncertainty in Measurement (GUM) [6]. Determining the range of uncertainty according to GUM often requires complex modeling of the error propagation or even Monte-Carlo Simulations, if an analytical expression cannot be given [7] or the influence factors, called input quantities, are not directly measurable. Therefore, applicable practical modeling techniques have been developed [8].

This paper presents a scalable linear uncertainty model according to the GUM procedures for measurements of room acoustic parameters. The main input quantities are determined by theoretical and experimental investigations of the measurement signal chain and its inner components. Special experiments are designed to be conducted in different types of halls, to investigate the dependence of the individual uncertainty contributions on the type and shape of the room. The combined uncertainty of the room acoustic parameter is calculated in a following step from the available data from the experiments. The uncertainty budget will be given and discussed in the context of measurement quality.

\section{Theoretical background}

In order to provide the necessary acoustic and mathematical background, the most important facts of the measurement standard for room acoustics (ISO 3382), which is the central document for room acoustic measurements, and the GUM, are summarized below. Knowledge of signal theory and of the correspondences between time and frequency domain (e.g. Fourier-transformation) are presumed.

\subsection{ISO 3382 - measurement standard}

ISO 3382 defines measurable objective acoustic parameters and their measurement procedures. These parameters have been designed to be related to human perception. For instance, the reverberation time $T$ corresponds to the perceived reverberance of a room and the clarity index $C_{80}$ is related to subjective sensation of clarity. Further parameters contain information on the spatial distribution of sound incidence at a listener's seat, e.g. Lateral Fraction. For demonstration purposes, this paper focuses on the clarity index, but the model is meant to be used for other acoustic parameters as well. To provide more detailed objective information at a certain position in a room, the parameters are required to be stated as a function of frequency by means of octave or third-octave frequency band values. The typical range covers octave bands from $63 \mathrm{~Hz}$ up to $8 \mathrm{kHz}$.

The object to be measured is the room, which can be assumed as a linear time-invariant (LTI) system, at least for the short time frame of a measurement session. Therefore, the impulse response completely describes the behavior of a room from a certain source to a certain receiver position in a system-theoretical manner. Acoustic parameters are defined as mathematical equations involving the impulse response $h(t)$. Hence, the effort is reduced to measurement of the impulse response only. The clarity index, which is representatively chosen, is defined by

$$
C_{\tau_{e}}=10 \log _{10}\left(\frac{\int_{0}^{\tau_{e}} h^{2}(t) \mathrm{d} t}{\int_{\tau_{e}}^{\infty} h^{2}(t) \mathrm{d} t}\right)[\mathrm{dB}]
$$

with an integration time $\tau_{e}=80 \mathrm{~ms}$ for music. This gives a relation between early and late arriving sound energy.

Besides the electronic equipment, e.g. PC and amplifiers, the essential components of the signal chain, as depicted in 


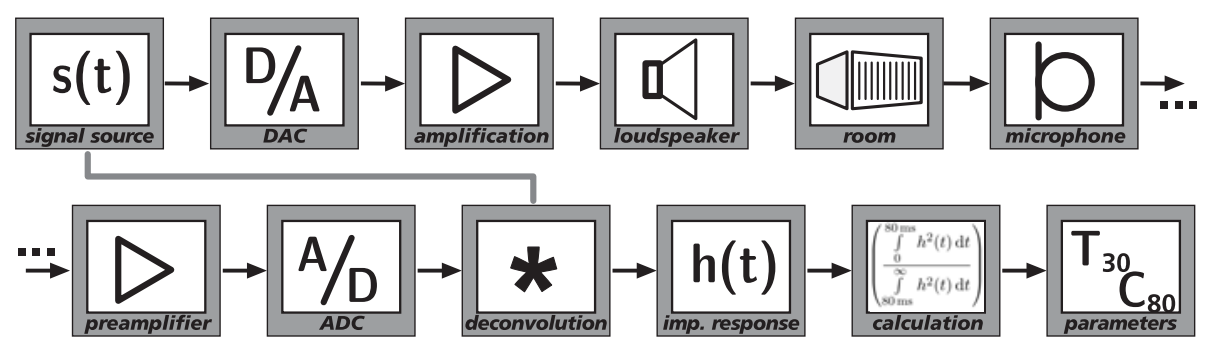

Fig. 1: Measurement chain in room acoustic measurements

Fig. 1, are the loudspeaker and the microphone, which are in direct interaction with the sound field of the room. For most room acoustic measurements a loudspeaker and microphones with omni-directional directivity patterns are required, as shown in Fig. 2. A typical geometrical setup for an omni-directional sound source is the dodecahedron loudspeaker, consisting of 12 equal loudspeaker chassis. The loudspeaker in Fig. 1 has been developed and enhanced at the Institute of Technical Acoustics at RWTH Aachen in recent years. It consists, from top to bottom, of a small high-tone dodecahedron, a mid-tone dodecahedron, and a bandpass sub-woofer speaker.
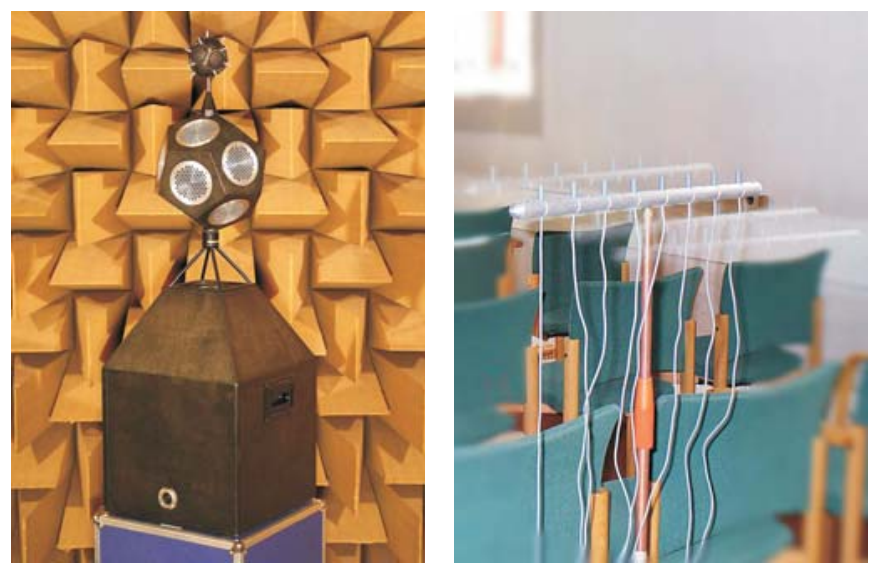

Fig. 2: 3-way dodecahedron loudspeaker (left) and condenser microphones in an array (right)

In addition, the measurement standard summarizes the investigated difference limen (jnd) for the defined room acoustic parameters. For the reverberation time the jnd is on the order of $5 \%$ of the mean reverberation time for each frequency band. For the clarity index it is assumed to be on the order of $1 \mathrm{~dB}$ independent of frequency.

\subsection{GUM basics}

The GUM was published 1995. It enhances the well known simple Gaussian error calculus by distinguishing between Type $A$ and Type $B$ uncertainty contributions instead of the formerly known random and systematic errors. In more detail, Type A covers results obtained by statistical analysis of a series of measurements and Type $B$ represents other analysis methods, e.g. using data from calibration certificates. The uncertainty of a measurement is defined as a parameter associated with a measurement result, called the measurand. It expresses a range of values which can reasonably be attributed to the measurand with a certain level of confidence. Typically, confidence levels of $68 \%$ and $95 \%$ are used, meaning that the true value lies in the given interval with this probability. Furthermore, the GUM describes a guideline scheme for deriving uncertainties, which consists of the following 7 parts.

1. Collecting information on the measurement and its input quantities $x_{i}$.

2. Modeling the particular measurement in terms of a model function $f$.

3. Evaluation of the input quantities according to Type $A$ or Type B.

4. Combination of the results to obtain the value $y$ and the associated uncertainty $u(y)$.

5. Calculating the expanded uncertainty $U(y)$.

6. Statement of the complete measurement result $y \pm U$ for a chosen coverage factor $k$.

7. Assembling the measurement uncertainty budget.

Formally, the output quantity $y$, which is the result of a measurement, can be expressed as a function of the input quantities $x_{i}$ as in the following

$$
y=f\left(x_{1}, x_{2}, \ldots, x_{N}\right) .
$$

If all input quantities $x_{i}$ are known, the corresponding uncertainties $u\left(x_{i}\right)$ have to be determined. In the case of Type $A$, which is used throughout this paper, the uncertainty can be expressed as the standard deviation of the mean

$$
u^{2}\left(x_{i}\right)=\frac{1}{n(n-1)} \sum_{k=1}^{n}\left(q_{k}-\bar{q}\right)^{2},
$$

where $q_{k}$ stands for the individual measurement results. The best value for the input quantity is defined as the arithmetic mean. Under consideration of these uncertainty contributions the combined uncertainty for the output quantity can be calculated as

$u(y)=\sqrt{\sum_{i=1}^{N}\left(\frac{\partial f}{\partial x_{i}} u\left(x_{i}\right)\right)^{2}+2 \sum_{i=1}^{N-1} \sum_{j=i+1}^{N} \frac{\partial f}{\partial x_{i}} \frac{\partial f}{\partial x_{j}} u\left(x_{i}, x_{j}\right)}$.

In most cases the correlation between the input quantities represented by $u\left(x_{i}, x_{j}\right)$ can be neglected.

\section{Application to room acoustics}

As mentioned above, determining the uncertainty contributions and the model function is the most difficult task in modeling uncertainties. Hence, certain assumptions and simplifications are made to apply the GUM procedures. As can be seen, the central measured quantity in room acoustics is the impulse response. Modeling the error propagation from the input quantities to the impulse response and for- 


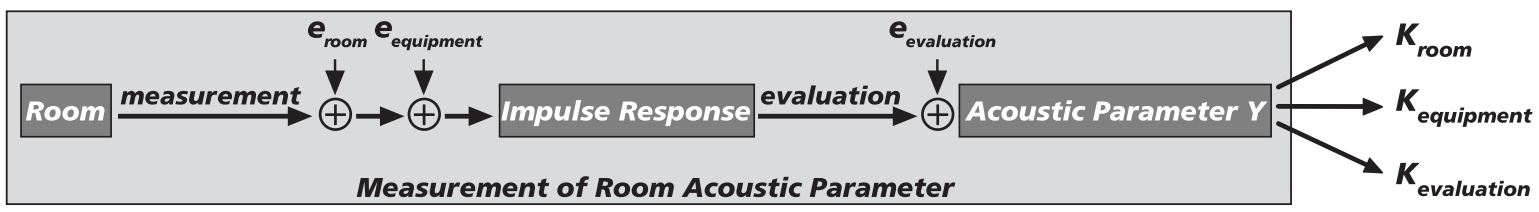

Fig. 3: Capsuled sources of measurement errors

ward to the derived parameters, involves finding a model function $f$ as in Equation (2). This is problematic, since the only input quantity for calculating a room acoustic parameter is the impulse response, which is not suitable in this context. In addition, the input quantities to the impulse response cannot always be directly measured.

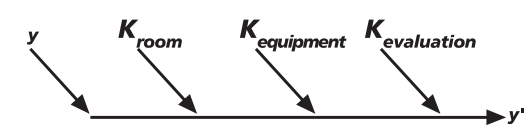

Fig. 4: Linear uncertainty dependence graph

As major uncertainty contributions can be found, it is applicable to analyze the variations of the output quantity itself by varying these input quantities. Fig. 3 illustrates this approach, where sources of errors are grouped into room, equipment and evaluation errors. The linear dependence graph in Fig. 4 shows that the corrected output value can be formulated as

$$
y=y^{\prime}-K_{\text {room }}-K_{\text {equipment }}-K_{\text {evaluation }}
$$

with the correction factors $K_{i}$. The model function $f$ is therefore linearized. Correction factors are introduced to capture the uncertainty contributions by means of a standard deviation of the output quantity. These are meant to have a mean value of $\overline{K_{i}}=0$ but an uncertainty $u\left(K_{i}\right) \neq 0$, modeling the different sources of error. As acoustic parameters are dependent on variables, e.g., frequency or position, the correction factors are generally assumed to be dependent on these variables until otherwise proven,

$$
K_{i}=K_{i}(f, \text { room, position })
$$

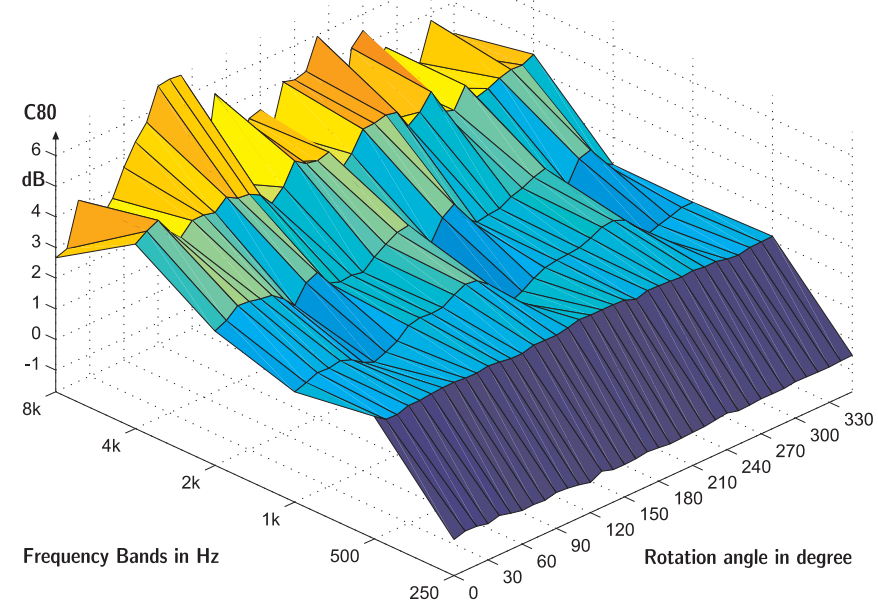

In more detail, Fig. 5 shows the grouping of the main input quantities influencing the accuracy of a room acoustic measurement. Hence, the task changes to finding experiments modeling the uncertainty of the input quantities in an appropriate manner and analyzing the output quantity, in terms of a correction factor, directly.

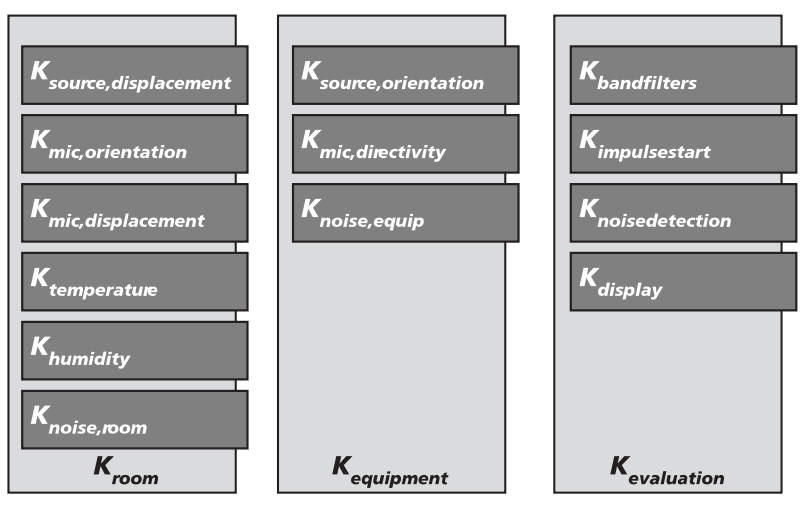

Fig. 5: Groups of correction factors with details

\section{Measurement Results}

Special experiments were designed to investigate, e.g., noise and meteorological influences, loudspeaker directivity or variations in positioning the microphones and the loudspeakers. Repeated overnight measurements were conducted, the dodecahedron loudspeaker was rotated by a controlled turntable, and the microphones were configured in an array to scan the area of a seat. The corresponding uncertainties are calculated from the standard deviations in each experiment for each frequency band independently.

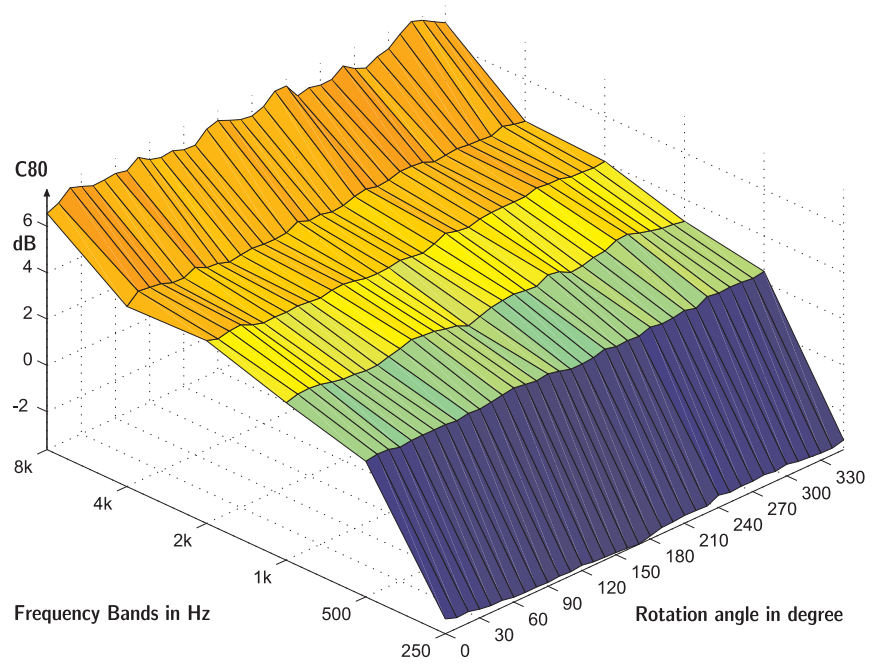

Fig. 6: Source rotation with two different dodecahedron loudspeakers 


$$
\mathrm{C}_{80} @ 250 \mathrm{~Hz}
$$

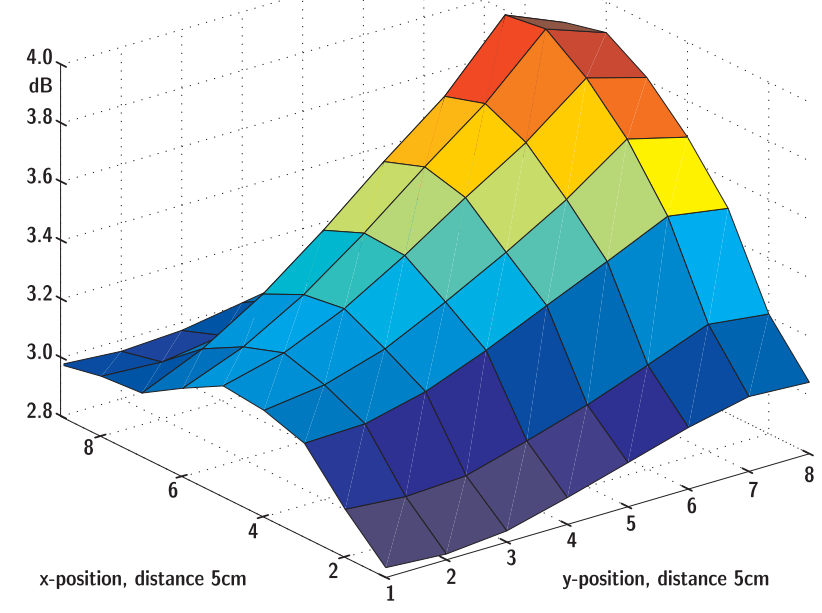

\section{$\mathrm{C}_{80} @ 500 \mathrm{~Hz}$}

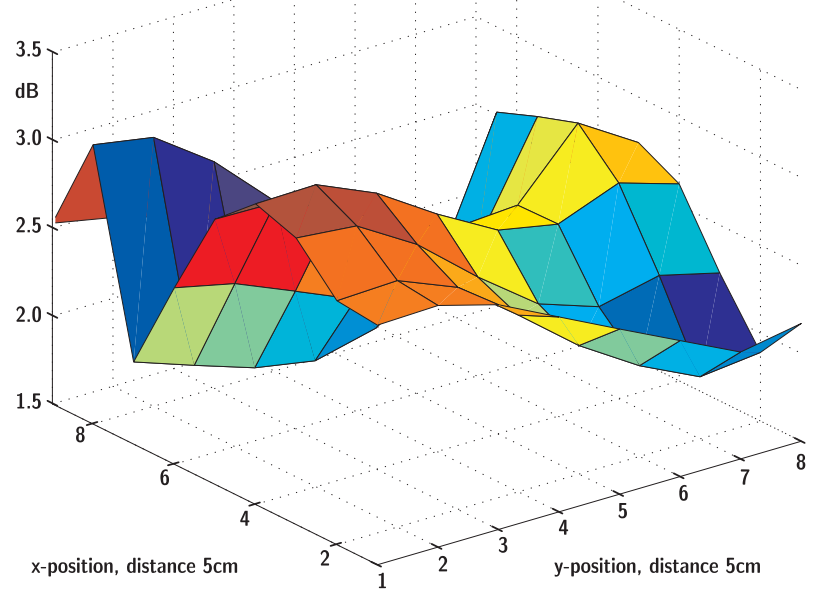

Fig. 7: Spatial variations within a seat position for two different frequency bands

Since the directional pattern of the dodecahedron loudspeaker has been under investigation at the Institute for Technical Acoustics for many years, measurement results obtained from two different loudspeakers on a turntable are presented to show the influence of directional pattern on the acoustic parameters. If the emitted wavelength is smaller than the geometry of the speaker, even the designed omni-directional source becomes more and more directional. Fig. 6 shows that the deviations in the calculated parameters increase with frequency for both sources used. In Fig. 6 (left), we use a commercially available dodecahedron loudspeaker commonly used for room acoustic measurements, and, in Fig. 6 (right), a special three-way dodecahedron loudspeaker with a smaller diameter, developed by the Institute of Technical Acoustics in Aachen. The results for the latter equipment show less variation, as the source is closer to omni-directionality. Due to its geometrical properties, the dodecahedron loudspeaker always shows a periodicity of $120^{\circ}$, as can be seen in the derived parameters.

The spatial distribution of the parameters within a seat, by means of an area in the seat at the typical height of the listeners' ears of $120 \mathrm{~cm}$ high above the floor, is drawn in Fig. 7.

Fig. 7 (left) shows the $250 \mathrm{~Hz}$ and Fig. 7 (right) the $500 \mathrm{~Hz}$ octave band. As can be seen, the parameters are dependent on the position and the fluctuations are in good agreement with the mean wavelength in these frequency bands. Therefore, the parameter varies faster over a position in the $500 \mathrm{~Hz}$ band than in the lower band. Towards higher frequencies, the variations decrease since the room's behavior changes from distinct room modes to overlapping modes. This transition happens at the well known Schröder frequency. By considering the measurement of a single seat position, the actual receiver one chooses when measuring a room could lay in the range of the scanned area. Therefore, it is applicable to adapt this fact to the uncertainty budget for octave bands, separately.

Further experiments and results have been obtained that are not discussed in detail here. The uncertainty budget under the given simplifications and assumptions can be seen in Fig. 8 for the major uncertainty contributions investigated. The combined uncertainty shown here is valid for measuring the room impulse response for one source and one seat position at a single time and deriving the room acoustic parameter. In this case, the uncertainty exceeds the difference limen. In order to provide more precise results, several measurements under variation of the source position and angle, the receiver position, etc., have to be averaged.

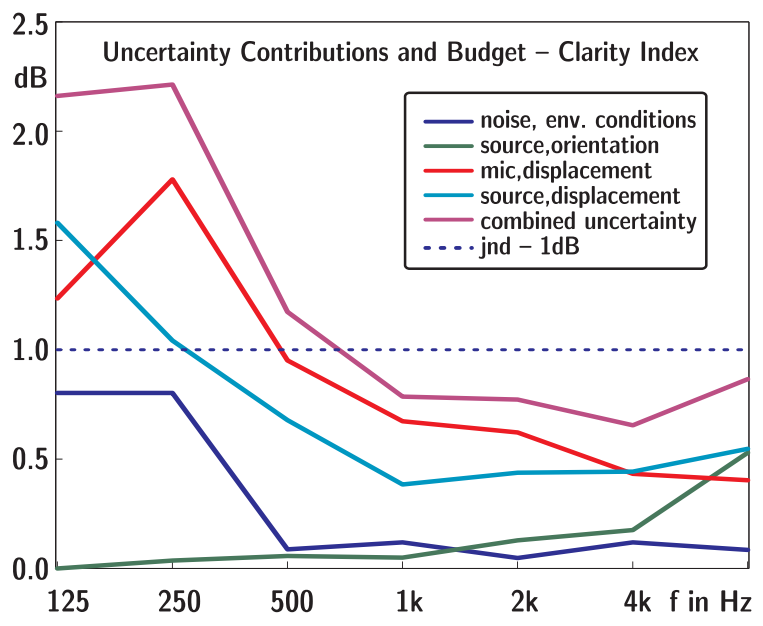

Fig. 8: Uncertainty budget for the clarity index

\section{Conclusion}

This paper has proposed an approach for modeling and determining uncertainty contributions in room acoustics, according to the GUM procedures, involving in-situ measurement results. Based upon the current measurement standard ISO 3382, the common measurement chain has been under uncertainty investigation. The main influence factors and their relevance regarding measurement uncertainty have been analyzed and determined. Experiments have been designed and conducted in several rooms and halls to quantify the uncertainty contributions and their dependence on different types of rooms. In a following step, the combined uncertainty for the output quantity was calculated and presented for the clarity index, representatively. However, the model, the procedures and the measured room impulse responses serve for uncertainty calculation of the remaining 
room acoustic parameters. The uncertainty budget for a single measurement shows values on the order of jnd. Hence, a single measurement for one source-receiver pair is not sufficient. Instead, several measurement results have to be used to give an average result.

\section{Acknowledgments}

The author would like to thank Prof. Michael Vorländer, Ingo Witew and Dr. Gottfried Behler from the Institute of Technical Acoustics at RWTH Aachen for their support. Furthermore, he would like to thank Mr. Ernsting, Mr. Mörtel, and Mr. Tennhard for providing the halls for measurement purposes.

\section{References}

[1] Bradley, J. S.: Using ISO 3382 measures, and their extensions, to evaluate acoustical conditions in concert halls. Acoustical science and technology, 2005.

[2] ISO, ISO 3382 - Acoustics - Measurement of the Reverberation Time - Part 1: Performance Spaces, ISO TC 43/SC 2 N 0767 - 2004-06-30, ISO, June 2004.

[3] ISO / DIS 18233, Acoustics - Application of New Measuring Methods in Building and Room Acoustics - Final Draft, ISO, 2006.
[4] Witew, I. B., Behler, G. K.: Uncertainties in Measurement of Single Number Parameters in Room Acoustics. Forum Acusticum, 2005.

[5] Lundeby, A., Vigran, T. E., Bietz, H.,Vorländer, M.: Uncertainties of Measurements in Room Acoustics. Acustica, Vol. 81 (1995), p. 344-355.

[6] Guide to the Expression of Uncertainty in Measurement. International Organization for Standardization, Geneva, 1995.

[7] Siebert, B. R. L., Sommer, K. D.: Weiterentwicklung des GUM und Monte-Carlo-Techniken. Technisches Messen, Vol. 71 (2004).

[8] Sommer, K. D., Weckenmann, A., Siebert, B. R. L.: A Systematic Approach to the Modelling of Measurements for Uncertainty Evaluation. Journal of Physics: Conference Series, Vol. 13 (2005), p. 224-227.

Dipl. Ing. Pascal Dietrich e-mail:pdi@akustik.rwth-aachen.de

Institute of Technical Acoustics

RWTH Aachen University D-52056 Aachen, Germany 